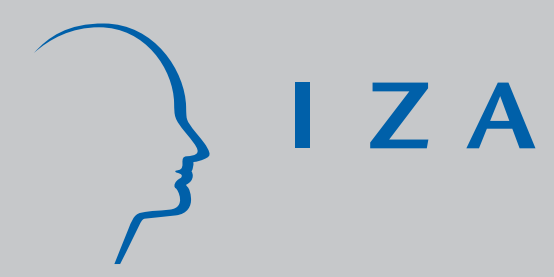

IZA DP No. 3671

Identification of Treatment Effects on the Treated with One-Sided Non-Compliance

Markus Frölich

Blaise Melly

August 2008 


\title{
Identification of Treatment Effects on the Treated with One-Sided Non-Compliance
}

\author{
Markus Frölich \\ University of Mannheim \\ and IZA \\ Blaise Melly \\ Brown University \\ Discussion Paper No. 3671 \\ August 2008 \\ IZA \\ P.O. Box 7240 \\ 53072 Bonn \\ Germany \\ Phone: +49-228-3894-0 \\ Fax: +49-228-3894-180 \\ E-mail: iza@iza.org
}

Any opinions expressed here are those of the author(s) and not those of IZA. Research published in this series may include views on policy, but the institute itself takes no institutional policy positions.

The Institute for the Study of Labor (IZA) in Bonn is a local and virtual international research center and a place of communication between science, politics and business. IZA is an independent nonprofit organization supported by Deutsche Post World Net. The center is associated with the University of Bonn and offers a stimulating research environment through its international network, workshops and conferences, data service, project support, research visits and doctoral program. IZA engages in (i) original and internationally competitive research in all fields of labor economics, (ii) development of policy concepts, and (iii) dissemination of research results and concepts to the interested public.

IZA Discussion Papers often represent preliminary work and are circulated to encourage discussion. Citation of such a paper should account for its provisional character. A revised version may be available directly from the author. 
IZA Discussion Paper No. 3671

August 2008

\section{ABSTRACT \\ Identification of Treatment Effects on the Treated with One-Sided Non-Compliance}

Traditional instrumental variable estimators do not generally estimate effects for the treated population but for the unobserved population of compliers. They do identify effects for the treated when there is one-sided perfect non-compliance. However, this property is lost when covariates are included in the model. In this case, we show that the effects for the treated are still identified but require modified estimators. We consider both average and quantile treatment effects and allow the instrument to be discrete or continuous.

JEL Classification: C13, C14, C21

Keywords: treatment effects, instrumental variables, non-compliance, missing data

Corresponding author:

Markus Frölich

Universität Mannheim

Abteilung Volkswirtschaftslehre

L7, 3-5

68131 Mannheim

Germany

E-mail: froelich@uni-mannheim.de 


\section{Introduction}

Instrumental variable estimators of a binary endogenous treatment generally identify only the treatment effect on the subpopulation of compliers (which is known as the local average treatment effect, LATE, Imbens and Angrist (1994) or the complier average causal effect CACE). LATE is the average effect for individuals whose treatment status is influenced by changing the value of the instrument. ${ }^{1}$ In many situations, however, we would rather like to know the effects on the treated, e.g. the average effect on the treated (ATET).

The population of compliers is not always of most interest. It is an unobservable population because we observe the value of the treatment only for one value of the instrument. This makes the interpretation of the findings less intuitive and can also limit their usefulness. For instance, Manski (2003) notes that findings for unobserved populations cannot be used by a planner to make treatment choice. In addition, for cost-benefit calculations we need to measure the impacts on those who actually have been treated. While we have individual data to estimate the benefits, we observe only the total cost of the program. To perform the cost-benefit analysis the treatment effects on the treated are required and not the complier average effects.

Out of these reasons, we would like to identify the effects for the treated population, e.g. the average effect (ATET) or quantile treatment effects on the treated (QTET). It is well known that in the case of one-sided non-compliance, the population of treated and compliers are identical such that the instrumental variables estimator estimates effects for the treated. However, as we argue in this note, if additional control variables are included in the model, treated and compliers are not identical. Nevertheless, the treatment effects on the treated are still identified, but different estimators have to be used. Our results also apply, by obvious modifications, to the regression discontinuity design (RDD), where one-sided noncompliance often occurs by design, see Battistin and Rettore (2008), who refer to this as a partially fuzzy design.

In the following we give a number of examples where this situation applies. In the first type of examples, treatment assignment is randomized, but the assignment probability differs between individuals. In this case, we have to control for the covariates that determined the assignment probability. Angrist (2006) examines a domestic violence experiment, where offenders were randomly subjected to one of three treatments: "arrest, ordering the offender off the premises

\footnotetext{
${ }^{1}$ The effects on quantiles or on other statistical characteristics of the marginal distributions of the outcome are also identified for the same population (e.g. Abadie, Angrist, and Imbens (2002) or Frölich and Melly (2007)).
} 
for eight hours, and some form of advice that might include mediation". One of the three treatments was randomly selected each time police officers encountered a situation of domestic assault. (Police officers could deviate from the random assignment and frequently did so.) Angrist (2006) mentions that the not-arresting "treatment might have been randomly assigned with higher probability to suspects with no prior history of assault. We then need to control for assault history in the IV analysis." Similarly, in clinical trials where assignment probability might depend on the severity of the illness. Finkelstein, Levin, and Robbins (1996) suggests a design where treatment assignment probability is $50 \%$ for everyone with severity of illness below a certain threshold and $100 \%$ for everyone above. ${ }^{2}$ Since the threshold is often arbitrary, a more effective approach is to gradually increase the assignment probability with the severity of illness (or other risk factors). Similarly, assignment probabilities often vary with publicly subsidized social programmes (e.g. training programmes) if local funding or supply constraints limit the number of places available (per region and per time period, e.g. each week or month). See e.g. Black, Galdo, and Smith (2005).

In the second type of examples, we consider randomized trials with missing outcomes. Nonresponse and attrition are universal problems of most randomized trials, particularly when one is interested in medium to long-term effects of a treatment. Assuming 'missing at random' MAR (conditional on covariates $X$ ) is the method of choice to deal with missingness (Little and Rubin 1987). ${ }^{3}$ (See e.g. Yau and Little (2001) on a randomized trial of job training assistance for unemployed.) As Frangakis and Rubin (1999) observe, this assumption might often be too strong. It can be relaxed by requiring ignorability to hold only conditionally on $X$ and the unobserved compliance type of the individual. Since the compliance type is unobserved, they also require an exclusion restriction for the never-taker. Mealli, Imbens, Ferro, and Biggeri (2004) examine a similar situation but rely on an exclusion restriction for compliers. In all these cases with missing data, the distribution of $X$ is not balanced among the populations with complete outcome data. We extend the methods of the mentioned articles by deriving estimators for unconditional treatment effects on the treated. ${ }^{4}$ We emphasize that our results straightforwardly generalize to the regression discontinuity design (RDD), where missing data has not been analyzed

\footnotetext{
${ }^{2}$ This allows for a RDD at the threshold.

${ }^{3}$ Apart from the rare cases where one has access to good instrumental variables for non-response.

${ }^{4}$ Missing outcome data also often occurs if for cost reasons we sample only a subset of the treated and controls and oversample high-risk groups, e.g. if we are interested in rare events, such as death.
} 
in the literature so far.

As a third example, we often like to include additional covariates $X$ to separate direct from indirect effects as in the spirit of Pearl (2000). ${ }^{5}$ A classic example in Pearl (2000) is the effect of taking the birth-pill on thrombosis. Taking the pill $(D)$ is suspected to increase the risk of thrombosis $(Y)$. At the same time does $D$ reduce the number of pregnancies, which are known to increase the risk of thrombosis. Here, we are not interested in the total effect, but only in the partial effect of $D$ on $Y$, i.e. which is not channeled via $X$. Similar ideas apply to the widely used Mincer earnings regressions, see e.g. Heckman, Lochner, and Todd (2004). We might be interested in the effects of class size on wages, and assign children randomly to large and small classes. The total effect is identified by the experimental design, but to obtain the partial effects one often includes labour market experience or profession/industry as a covariate in the regression.

Finally, we mention the situation where the instrumental variable has not been randomly assigned and therefore might be confounded, unless we condition on several background characteristics. To give just one example, Pitt and Khandker (1998) examine the effects of microcredit on various labour market and health outcomes. Households with more than half an acre of land, however, are not eligible to participate in these microcredit programmes. Let $Z=1$ if landholdings are below half an acre, and $Z=0$ otherwise. Some households with $Z=1$ receive microcredit $(D=1)$ while others do not $(D=0)$. At the same time, landholdings $Z$ are likely to be correlated with other background variables $X$ that might have an independent effect on various outcome variables, e.g. health outcomes, and thus need to be conditioned on.

Section 2 presents the framework and discusses the (trivial) case without covariates. We show in section 3 that the treatment effects are also identified in the presence of covariates. Sub-section 3.1 gives a regression (matching) representation of the effects while section 3.2 presents weighted representations of the estimands. In both cases we allow for continuous or discrete instruments. Finally, section 4 presents results for missing outcomes.

\section{Instrumental variables and one-sided non-compliance}

Consider a binary treatment $D_{i} \in\{0,1\}$ and let $Y_{i}^{1}, Y_{i}^{0}$ be the potential outcomes in case of treatment and non-treatment, respectively. In the medical drug trial example, the treatment

\footnotetext{
${ }^{5}$ We should be aware that including such post-treatment variables might introduce bias if those are correlated with unobservables affecting the outcome variable.
} 
choice is $D_{i} \in\{$ no drug,drug $\}$ and the outcome of interest is blood pressure one week after treatment. Let $Z_{i}$ be an instrumental variable that has an effect on $D_{i}$ but no direct effect on the potential outcomes. For example, $Z$ could be random assignment to treatment versus no-treatment, but later we also allow for other, not necessarily binary, instruments.

With random assignment of $Z$, the intention to treat effect (ITT) is immediately identified by regressing $Y$ on a constant and $Z$. Of most interest, however, is not the ITT but rather the treatment effect of $D$ on $Y$, either the average treatment effect $E\left[Y^{1}-Y^{0}\right]$ or in particular the average treatment effect on the treated (ATET)

$$
E\left[Y^{1}-Y^{0} \mid D=1\right]
$$

which receives most attention in the evaluation literature. The ATET refers to the subpopulation, which actually received the treatment and thus realized the impact of the treatment and is therefore the appropriate subpopulation for a cost-benefit analysis.

If everyone complied with the random assignment, then $D=Z$ and ITT=ATE=ATET. However, in many applications the experimental protocol is violated. Non-compliance can be in two directions: Individuals assigned to treatment $\left(Z_{i}=1\right)$ may not take the treatment $\left(D_{i}=0\right)$ and, vice versa, individuals assigned to non-treatment $\left(Z_{i}=0\right)$ may receive the treatment $\left(D_{i}=\right.$ 1). In many applications, however, only one-sided non-compliance occurs. For example, if a new medical drug is tested, it is impossible for someone assigned to placebo $\left(Z_{i}=0\right)$ to gain access to the new drug. Still, those who are assigned to treatment may not take the drug. Similarly with participation in a training programme. Individuals assigned to training may not attend the course. On the other hand, individuals randomized out may not be able or willing to take part in the course, e.g. if the course providers are not permitted to accept them or charge very high fees.

With one-sided non-compliance, we can distinguish two different types of individuals: Those who would take the treatment if assigned to it, and those who would not take the treatment irrespective of $Z .{ }^{6}$ We will call the former group the "compliants" or "compliers" and the latter group the "never treated". As shown in Imbens and Angrist (1994), the average treatment effect on the compliers, called LATE, can be identified by instrumental variable estimation. Because with one-sided non-compliance and random assignment of $Z$, only the compliers are those who

\footnotetext{
${ }^{6}$ If the one-sided non-compliance is in the other direction, redefine $D$ as $1-D$. Of course, in this case we would identify only effects for the non-treated.
} 
receive the treatment, it follows that $\mathrm{LATE}=\mathrm{ATET}$ such that

$$
E\left[Y^{1}-Y^{0} \mid D=1\right]=\frac{E[Y \mid Z=1]-E[Y \mid Z=0]}{E[D \mid Z=1]}
$$

see also Bloom, Orr, Bell, Cave, Doolittle, Lin, and Bos (1997). By straightforward modifications we can also obtain the quantile treatment effects for the treated (QTET). Hence, the ATET and QTET are identified.

The situation is different when we include additional control variables. Several reasons for doing so have been discussed in the introduction. In this case, the populations of treated and of compliers have different $X$ distributions such that ATET $\neq$ LATE. More precisely, the ATET corresponds to the treatment effect on the treated compliers, which can be different from LATE.

\section{Identification of treatment effects for the treated}

We still consider the case where $D$ is binary, but permit $Z$ and $X$ to be of any dimension and any type. If $Z$ has a mass point at $z_{0}, f_{Z}\left(z_{0}\right)=\operatorname{Pr}\left(Z=z_{0}\right)$; if $Z$ is continuous, $f_{Z}\left(z_{0}\right)$ denotes the probability density function. We assume in the following:

\section{Assumption 1:}

i) One-sided non-compliance: $\quad \operatorname{Pr}\left(D=0 \mid Z=z_{0}\right)=1$

ii) Existence of compliers: $\quad \operatorname{Pr}(D=1)>0$

iii) Independent instrument: $\quad Y^{0} \Perp Z \mid X$

iv) Support condition: $\quad f_{Z \mid X}\left(z_{0}\right)>0 \quad$ for almost every $X$

Assumption (i) is the one-sided perfect compliance assumption that is central in this paper. Assumption (ii) requires that the instruments have some power in that there are at least some individuals who react to it. The third assumption is the main instrumental variable assumption. The fourth assumption requires that $Z=z_{0}$ may be observed given almost all values of $X$. Assumption (i) has no identifying power if $Z=z_{0}$ cannot be observed. ${ }^{7}$ This assumption also

\footnotetext{
${ }^{7}$ If $Z$ has a mass-point at $z_{0}$ this assumption requires that $\operatorname{Pr}\left(Z=z_{0} \mid X\right)>0$. If $Z$ is continuous, some additional technical regularity conditions should be added (either smoothness of $f_{Z \mid X}(z)$ at $z_{0}$ or of the conditional distribution of $Y$ given $X$ and $Z$ at $z_{0}$, compactness of the support of $Z$ ) in order to ensure the consistency of potential estimators. We do not further discuss these technical details in the following.
} 
covers the assumption of Imbens and Angrist (1994) but is weaker in many respects, apart from the one-sided non-compliance assumption.

We need one point $z_{0}$ where receipt of treatment is impossible. E.g. in the medical trial example, $Z$ is binary and at $z_{0}=0$ we have $\operatorname{Pr}\left(D=1 \mid Z=z_{0}\right)=0$, i.e. those assigned placebo cannot get access to the new drug. We embrace a more general setup here where $Z$ can be nonbinary. E.g. $Z$ may represent different intensities with which patients in the treatment group are encouraged or given incentives to take the prescribed drug. (In the placebo group, $Z=0$.) We thus permit non-binary $Z$, do not require monotonicity and only need the instrumental variable condition to hold conditional on $X$. Note that for identification of ATET, Assumption 1(iii) could be weakened to require only mean independence, but for identification of quantile treatment effects, full independence is required.

\subsection{Regression representation}

We will show in this section that ATET and QTET are identified by Assumption 1. In order to provide succinctly the results for ATET, QTET and any other statistic based on moments of the marginal distribution of $Y_{0}$, Theorem 1 states a general result that will be specialized later to the cases of ATET and QTET.

Theorem 1 (Regression) Let $g(\cdot)$ be any real measurable function such that $E\left|g\left(Y^{0}\right)\right|<\infty$. Under Assumption 1

$$
E\left[g\left(Y^{0}\right) \mid D=1\right]=\frac{\int E\left[g(Y) \mid X, Z=z_{0}\right] d F_{X}-E[g(Y) \cdot(1-D)]}{\operatorname{Pr}(D=1)}
$$

Theorem 1 is a powerful identification result. It says that any statistical characteristic that can be defined in terms of moments of the distribution of $Y^{0}$ is identified for the treated. Making use of this result we obtain after a few calculations a simple representation for the ATET:

Corollary 2 Under Assumption 1

$$
E\left[Y^{1}-Y^{0} \mid D=1\right]=\int \frac{E[Y \mid X]-E\left[Y \mid X, Z=z_{0}\right]}{\operatorname{Pr}(D=1)} d F_{X}=\frac{E[Y]-\int E\left[Y \mid X, Z=z_{0}\right] d F_{X}}{\operatorname{Pr}(D=1)} .
$$


Note that this simplifies to equation (1) if $X$ is the empty set and if $Z$ is binary. A natural estimator for the ATET is obtained by plugging in nonparametric regression estimators of $E[Y \mid X, Z]$ and $E[Y \mid X]$ to estimate ATET as

$$
\frac{\sum_{i=1}^{n}\left(Y_{i}-\hat{E}\left[Y \mid X_{i}, Z=z_{0}\right]\right)}{\sum_{i=1}^{n} D_{i}} .
$$

Note that this formula is very different from LATE, which has been derived in Frölich (2007):

$$
E\left[Y^{1}-Y^{0} \mid \mathcal{T}=\text { complier }\right]=\frac{\int\left(E\left[Y \mid X, Z=z_{H}\right]-E\left[Y \mid X, Z=z_{0}\right]\right) \cdot d F_{X}}{\int\left(E\left[D \mid X, Z=z_{H}\right]-E\left[D \mid X, Z=z_{0}\right]\right) \cdot d F_{X}},
$$

where $z_{H}=\underset{z \in \operatorname{Supp}(Z)}{\arg \max } \operatorname{Pr}(D=1 \mid Z=z)$ is the value of the instrument which induces the largest fraction of people to treatment, and where we have to assume monotonicity additionally.

In many research areas, one is not only interested in the average treatment effect, but also in its distributional effects. Consider two different training programmes with the same (positive) average effect on wages. If the impacts of the first programme are mostly be found in the lower tail of the wage distribution, whereas the second programme impacts on the upper tail, policy makers would probably favour the former programme. Another example which has received considerable public interest is educational equality, where many societies would prefer to provide every child with a fair chance into adult live. Here, $Y$ is a measure of cognitive ability (e.g. obtained from math and language tests) and $D$ may be the introduction of computers in classroom (teaching). Quantile treatment effects (QTE) are then appealing to characterize the heterogeneous impacts of the treatment at different points of the outcome distribution.

Frölich and Melly (2007) derived the quantile treatment effects for the compliers. Here we are interested in the quantile treatment effects on the treated (QTET). The QTET is defined as

$$
Q_{Y^{1} \mid D=1}^{\tau}-Q_{Y^{0} \mid D=1}^{\tau}
$$

The first part is trivially identified as the quantile of $Y$ in the $D=1$ population as this is the factual outcome. More difficult is the counterfactual outcome $Q_{Y^{0} \mid D=1}^{\tau}$. We first identify the distribution of the counterfactual outcome of the treated and then invert the distribution. 
Corollary 3 Under Assumption 1

$$
\begin{aligned}
Q T E T^{\tau} & =Q_{Y \mid D=1}^{\tau}-F_{Y^{0} \mid D=1}^{-1}(\tau) \text { with } \\
F_{Y^{0} \mid D=1}(u) & =\int \frac{E\left[1(Y \leq u) \mid X, Z=z_{0}\right]+E[1(Y \leq u) \cdot(D-1) \mid X]}{\operatorname{Pr}(D=1)} \cdot d F_{X} .
\end{aligned}
$$

Straightforward nonparametric estimators exist for all elements appearing in Corollary 2. An alternative that may be fruitful when we want to estimate the whole distribution (or at least for a large number of $u$ ) consists in estimating the conditional quantile function by local quantile regression and then to invert this function. Instead of using kernel weights, nearest neighbors estimators may also be used to estimate all conditional functions.

\subsection{Weighting representation}

The identification result of Theorem 1 can be considered as a regression (or matching) representation of the estimands. Estimators based on it require the (nonparametric) estimation of the conditional expected value or conditional distribution of $Y$ as a function of $X$ and $Z$. Theorem 2 will show that an alternative weighted representation exists. The estimation of the weights requires the estimation of $f_{Z \mid X}\left(z_{0} \mid x\right)$. Therefore, estimators based on this representation are especially useful when this function is easier to estimate than the regression function, e.g. when $Z$ is randomized and we know the randomization function. It has also the advantage of providing an explicit representation for the counterfactual quantiles.

Theorem 4 (Weighting) Let $g(\cdot)$ be any real measurable function such that $E\left|g\left(Y^{0}\right)\right|<\infty$. Under Assumption 1

$$
E\left[g\left(Y^{0}\right) \mid D=1\right]=\frac{1}{\operatorname{Pr}(D=1)} E\left[g(Y) \cdot(1-D) \frac{1\left(Z=z_{0}\right)-f_{Z \mid X}\left(z_{0} \mid X\right)}{f_{Z \mid X}\left(z_{0} \mid X\right)}\right]
$$

In the case where the instrument is binary and $z_{0}=0$, the result of Theorem 2 specializes to

$$
E\left[g\left(Y^{0}\right) \mid D=1\right]=\frac{1}{\operatorname{Pr}(D=1)} E\left[g(Y) \cdot(1-D) \frac{\operatorname{Pr}(Z=1 \mid X)-Z}{1-\operatorname{Pr}(Z=1 \mid X)}\right] .
$$

This result can be used directly to obtain a weighted representation for the ATET: 
Corollary 5 Under Assumption 1

$$
E\left[Y^{1}-Y^{0} \mid D=1\right]=\frac{1}{\operatorname{Pr}(D=1)} E\left[Y \cdot\left(D-(1-D) \frac{1\left(Z=z_{0}\right)-f_{Z \mid X}\left(z_{0} \mid X\right)}{f_{Z \mid X}\left(z_{0} \mid X\right)}\right)\right]
$$

Theorem 2 can also be used to obtain a representation of the QTET as a difference between the solutions of two weighted quantiles

\section{Corollary 6 Under Assumption 1}

$$
\begin{aligned}
Q_{Y^{1} \mid D=1}^{\tau} & =\underset{q_{1}}{\arg \min } E\left[\rho_{\tau}\left(Y-q_{1}\right) \mid D=1\right] \\
Q_{Y^{0} \mid D=1}^{\tau} & =\underset{q_{0}}{\arg \min E}\left[\rho_{\tau}\left(Y-q_{0}\right) \cdot \frac{1\left(Z=z_{0}\right)-f_{Z \mid X}\left(z_{0} \mid X\right)}{f_{Z \mid X}\left(z_{0} \mid X\right)} \mid D=0\right]
\end{aligned}
$$

where $\rho_{\tau}(u)=u \cdot\{\tau-1(u<0)\}$.

The quantile $Q_{Y^{1} \mid D=1}^{\tau}$ can thus be obtained by a simple unweighted quantile regression on a constant in the $D=1$ subsample, whereas a weighted quantile regression on a constant in the $D=0$ subsample identifies $Q_{Y^{0} \mid D=1}^{\tau}$. Given a (nonparametric) estimate of $f_{Z \mid X}\left(z_{0} \mid x\right)$, natural estimators are

$$
\begin{aligned}
\hat{Q}_{Y^{1} \mid D=1}^{\tau} & =\underset{q_{1}}{\arg \min } \sum_{i: D_{i}=1} \rho_{\tau}\left(Y-q_{1}\right) \\
\hat{Q}_{Y^{0} \mid D=1}^{\tau} & =\underset{q_{0}}{\arg \min } \sum_{i: D_{i}=0} \rho_{\tau}\left(Y-q_{0}\right) \cdot \frac{1\left(Z=z_{0}\right)-\hat{f}_{Z \mid X}\left(z_{0} \mid x\right)}{\hat{f}_{Z \mid X}\left(z_{0} \mid x\right)} .
\end{aligned}
$$

Again, these estimators for QTET are different from the quantile treatment effect for all compliers, as derived in Frölich and Melly (2007). Asymptotic properties of the estimators of QTET could be derived by extending their results.

\section{Missing outcome data}

A particularly important case where including control variables even in a fully randomized trial is important relates to missing data. As Frangakis and Rubin (1999) observe "Randomised experiments with human subjects often suffer from two major complications, namely noncompliance to treatment assignment and missing outcomes". Different identification assumptions can 
be exploited, which in a first stage require conditioning on certain control variables. Finally, however, we are interested in the treatment effect on all treated, e.g. for a cost-benefit analysis. We will cover different sets of identifying assumptions, whose plausibility needs to be assessed in the particular application. For the sake of brevity, we will derive only the regression representation forms of the identified potential outcomes (as in Section 3.1), and note that weighting representations as in Section 3.2 could be derived similarly.

We will maintain throughout Assumption 1. Therefore, although we focus here on the missing data issue, we still permit that the assignment probability might vary with $X$. Suppose that data on $Z, X$ and $D$ is observed for all individuals, but the outcome variable $Y$ is observed only for some individuals. Let the response indicator $R_{i}=1$ if $Y_{i}$ is observed.

We examine first, the assumption of missingness at random conditional on all observed covariates:

$$
Y \Perp R \mid X, Z, D \text {. }
$$

Under this assumption we identify the potential outcomes for the treated as follows

Corollary 7 Under Assumptions 1 and (5), the potential outcomes for the treated are identified as

$$
\begin{aligned}
& E\left[g\left(Y^{0}\right) \mid D=1\right]=\int \frac{E\left[g(Y) \mid X, R=1, Z=z_{0}\right]-E[g(Y) \mid X, Z, R=1, D=0] \operatorname{Pr}(D=0 \mid X, Z)}{\operatorname{Pr}(D=1)} \cdot d F_{X, Z} \\
& E\left[g\left(Y^{1}\right) \mid D=1\right]=\int \frac{E[g(Y) \mid X, Z, R=1, D=1] \operatorname{Pr}(D=1 \mid X, Z)}{\operatorname{Pr}(D=1)} \cdot d F_{X, Z} .
\end{aligned}
$$

Note that this result is similar to Theorem 1, but the exact formula differs since the response indicator does not enter in all conditioning sets.

The MAR restriction (5) assumes that the response behaviour is a function of the received treatment $D$ (in addition to the covariates $X$ and $Z$ ). Receiving or not receiving the treatment impacts on the individuals' probability to respond. Frangakis and Rubin (1999) argued that in many studies it is not the treatment receipt that determines response behavior but rather the unobserved type of the individual. To relate ourselves to the common terminology in the literature, we define $D_{i, z}$ as the potential treatment status if $Z_{i}$ were hypothetically set to $z$. For any particular value of $z$, there will be two types of individuals: those with $D_{i, z}=0$ and those with $D_{i, z}=1$. If the instrumental variable is binary and we choose $z=1\left(z_{0}=0\right)$, the former group is usually referred to as the "never-takers" while the latter group is referred to as 
the "compliers". When $Z$ is non-binary, we can think of $D_{i, z}$ as defining compliance status when $Z$ takes the value $z$ vis-a-vis $z_{0}$.

We replace now the MAR assumption (5), which assumed independence conditional on the observed $D$, by the latent ignorability assumption of Frangakis and Rubin (1999), which requires independence conditional on the unobserved type $D_{z}$ :

For any value $z \in \operatorname{Supp}(Z)$

$$
Y \Perp R \mid X, Z, D_{z}
$$

Since the type is only revealed when $Z=z$ is observed, we need one further assumption to disentangle the response probabilities when $Z=z_{0}$. For the case when $Z$ is binary, Frangakis and Rubin (1999) assume that the response is independent of the instrument for the nevertakers. Since this population does not receive the treatment irrespective of the value of $Z$, it may be reasonable to assume that $Z$ also does not affect their response behaviour. On the other hand, we do not restrict the response behavior of the compliers, which is permitted to be dependent on $Z$. To permit for non-binary $Z$ we state this assumption of Frangakis and Rubin (1999) as:

For any value $z \in \operatorname{Supp}(Z)$

$$
Z \Perp R \mid X, D_{z}=0 .
$$

Corollary 8 Under assumptions 1, (6) and (7), the potential outcomes for the treated are identified as

$$
\begin{aligned}
E\left[g\left(Y^{0}\right) \mid D=1\right] & =\int \frac{E\left[g(Y) R \mid X, Z=z_{0}\right]-E[g(Y) R(1-D) \mid X, Z]}{E\left[R \mid X, Z=z_{0}\right]-E[R(1-D) \mid X, Z]} \cdot d F_{X, Z \mid D=1} \\
E\left[g\left(Y^{1}\right) \mid D=1\right] & =\int E[g(Y) \mid X, Z, R=1, D=1] \cdot d F_{X, Z \mid D=1} .
\end{aligned}
$$

Identification of the potential outcomes in the previous theorem relied on the ignorability of response for the never-takers (7), which is a plausible assumption particularly in double-blind studies. As an alternative, we can make this ignorability condition for the compliers. Mealli, Imbens, Ferro, and Biggeri (2004) observe that this latter assumption might be more plausible in certain circumstances. Although never-takers never receive the treatment irrespective of the value of $Z$ such that their outcomes are plausibly unaffected by $Z$, they do not comply with the treatment assignment. If the study is not double-blind, never-takers who happen to be 
assigned to $Z=1$ and decided to not comply with their assignment might have a different (perhaps lower) response probability than those never-takers who happen to be assigned to $Z=0$. The choice to refuse the active treatment if offered, might also compromise their willingness to participate in follow-up surveys. The compliers, on the other hand, are willing to comply with their treatment assignment and might thus be equally willing to provide follow-up data, irrespective of the treatment actually received. In this situation, (8) might be more plausible.

To permit for non-binary $Z$ we state this assumption as: For any value $z \neq z_{0} \in \operatorname{Supp}(Z)$

$$
Z \Perp R \mid X, D_{z}=1 .
$$

Corollary 9 Under assumptions 1, (6) and (8), the potential outcomes for the treated are identified as

$$
\begin{aligned}
& E\left[g\left(Y^{0}\right) \mid D=1\right] \\
&= \int \frac{E\left[g(Y) R \mid X, Z=z_{0}\right]-E[g(Y) \mid X, Z, R=1, D=0] \cdot\left(E\left[R \mid X, Z=z_{0}\right]-E[R D \mid X, Z]\right)}{E[R \mid X, Z, D=1] \operatorname{Pr}(D=1)} \cdot d F_{X, Z} \\
& E\left[g\left(Y^{1}\right) \mid D=1\right]=\int E[g(Y) \mid X, Z, R=1, D=1] \cdot d F_{X, Z \mid D=1} .
\end{aligned}
$$

\section{Conclusions}

In this note, we have shown that instrumental variables identify treatment effects not only for the compliers but also for the treated when there is only one-sided noncompliance. Without any control variables, ATET=LATE, but when control variables are included, the estimands differ. New estimators have been suggested that allow for the presence of continuous and discrete control variables and instruments. We also show identification of treatment effects on the treated in the presence of missing outcomes under three alternative sets of assumptions.

\section{References}

Abadie, A., J. Angrist, and G. Imbens (2002): "Instrumental Variables Estimates of the Effect of Subsidized Training on the Quantiles of Trainee Earnings," Econometrica, 70, 91117 .

ANGRIST, J. (2006): "Instrumental variables methods in experimental criminological research: what, why and how," Journal of Experimental Criminology, 2, 23-44. 
Battistin, E., and E. Rettore (2008): "Ineligibles and eligible non-participants as a double comparison group in regression-discontinuity designs," Journal of Econometrics, 142, 715-730.

Black, D., J. Galdo, and J. Smith (2005): "Evaluating the regression discontinuity design using experimental data," mimeo, University of Michigan, USA.

Bloom, H., L. Orr, S. Bell, G. Cave, F. Doolittle, W. Lin, and J. Bos (1997): "The Benefits and Costs of JTPA Title II-A Programs: Key Findings from the National Job Training Partnership Act Study," Journal of Human Resources, 32, 549-576.

Finkelstein, M., B. Levin, and H. Robbins (1996): "Clinical and Prophylactic Trials with Assured New Treatment for Those at Greater Risk: I. A Design Proposal," American Journal of Public Health, 86, 691-695.

Frangakis, C., And D. Rubin (1999): "Addressing complications of intention-to-treat analysis in the combined presence of all-or-none treatment-noncompliance and subsequent missing outcomes," Biometrika, 86, 365-379.

FrÖlich, M. (2007): "Nonparametric IV Estimation of Local Average Treatment Effects with Covariates," Journal of Econometrics, 139, 35-75.

FröLICH, M., AND B. Melly (2007): "Unconditional quantile treatment effects under endogeneity," IZA discussion paper, 3288.

Heckman, J., L. Lochner, and P. Todd (2004): "Fifty Years of Mincer Earnings Equations," mimeo, University of Chicago.

Imbens, G., And J. Angrist (1994): "Identification and Estimation of Local Average Treatment Effects," Econometrica, 62, 467-475.

Little, R., And D. Rubin (1987): Statistical Analysis with Missing Data. Wiley, New York.

Manski, C. F. (2003): Partial Identification of Probability Distributions. New York: Springer Verlag.

Mealli, F., G. Imbens, S. Ferro, and A. Biggeri (2004): "Analyzing a randomized trial on breast self-examination with noncompliance and missing outcomes," Biostatistics, 5, 207-222s.

Pearl, J. (2000): Causality: Models, Reasoning, and Inference. Cambridge University Press, Cambridge.

Pitt, M., And S. Khandker (1998): "The Impact of Group-Based Credit Programs on Poor Households in Bangladesh: Does the Gender of Participants Matter?," Journal of Political Economy, 106, 958-996.

YAU, L., And R. Little (2001): "Inference for the complier-average causal effect from longitudinal data subject to noncompliance and missing data, with application to a job training assessment for the unemployed," Journal of American Statistical Association, 96, 1232-1244. 


\section{A Appendix}

\section{A.1 Proof of Theorem 1}

As a preliminary, note that by Assumptions 1 (i) and (iii) for any $z$

$$
\begin{aligned}
E\left[g(Y) \mid X, Z=z_{0}\right]= & E\left[g\left(Y^{0}\right) \mid X, Z=z\right] \\
=E\left[g\left(Y^{0}\right) \mid X, Z=\right. & z, D=1] \cdot \operatorname{Pr}(D=1 \mid X, Z=z) \\
& +E\left[g\left(Y^{0}\right) \mid X, Z=z, D=0\right] \cdot \operatorname{Pr}(D=0 \mid X, Z=z)
\end{aligned}
$$

such that the counterfactual outcome, conditional on $X$, is identified as $E\left[g\left(Y^{0}\right) \mid X, Z=z, D=1\right]=\frac{E\left[g(Y) \mid X, Z=z_{0}\right]-E[g(Y) \mid X, Z=z, D=0] \cdot \operatorname{Pr}(D=0 \mid X, Z=z)}{\operatorname{Pr}(D=1 \mid X, Z=z)}$.

Now we examine the unconditional counterfactual outcome using Assumption 1(i) and (9)

$$
\begin{aligned}
& E\left[g\left(Y^{0}\right) \mid D=1\right] \\
= & \iint E\left[g\left(Y^{0}\right) \mid X=x, Z=z, D=1\right] \cdot d F_{Z \mid D, X}(z \mid 1, x) \cdot d F_{X \mid D}(x \mid 1) \\
= & \iint \frac{E\left[g(Y) \mid X=x, Z=z_{0}\right]-E[g(Y) \mid X=x, Z=z, D=0] \cdot \operatorname{Pr}(D=0 \mid X, Z=z)}{\operatorname{Pr}(D=1 \mid X, Z=z)} \\
& \cdot d F_{Z \mid D, X}(z \mid 1, x) \cdot d F_{X \mid D}(x \mid 1) \\
= & \iint \frac{E\left[g(Y) \mid X=x, Z=z_{0}\right]-E[g(Y) \cdot(1-D) \mid X=x, Z=z]}{\operatorname{Pr}(D=1)} \cdot d F_{Z \mid X}(z \mid x) \cdot d F_{X}(x) \\
= & \int \frac{E\left[g(Y) \mid X=x, Z=z_{0}\right]-E[g(Y) \cdot(1-D) \mid X=x]}{\operatorname{Pr}(D=1)} \cdot d F_{X}(x)
\end{aligned}
$$

\section{A.2 Proof of Corollary 2}

Apply Theorem 1 with $g\left(Y^{0}\right)=Y^{0}$

$$
\begin{aligned}
& E\left[Y^{1}-Y^{0} \mid D=1\right] \\
= & \int E[Y \mid X, D=1] \cdot d F_{X \mid D=1}-E\left[Y^{0} \mid D=1\right] \\
= & \int E[Y \mid X, D=1] \cdot \frac{\operatorname{Pr}(D=1 \mid X)}{\operatorname{Pr}(D=1)} \cdot d F_{X}-E\left[Y^{0} \mid D=1\right] \\
= & \int \frac{E[Y D \mid X]}{\operatorname{Pr}(D=1)} \cdot d F_{X}-E\left[Y^{0} \mid D=1\right]=\int \frac{E[Y \mid X]-E\left[Y \mid X, Z=z_{0}\right]}{\operatorname{Pr}(D=1)} d F_{X} .
\end{aligned}
$$

\section{A.3 Proof of Corollary 3}

Apply Theorem 1 with $g\left(Y^{0}\right)=1\left(Y^{0} \leq u\right)$. 


\section{A.4 Proof of Theorem 4}

Note that by iterated expectations

$$
\begin{aligned}
\int E\left[g(Y) \mid X, Z=z_{0}\right] d F_{X} & =\iint g(y) \cdot f_{Y \mid X, Z}\left(y \mid x, z_{0}\right) d y d F_{X}(x) \\
& =\iint g(y) \cdot \frac{f_{Y, Z \mid X}\left(y, z_{0} \mid x\right)}{f_{Z \mid X}\left(z_{0} \mid x\right)} d y d F_{X}(x) \\
& =\iiint g(y) \cdot \frac{1\left(Z=z_{0}\right) \cdot f_{Y, Z \mid X}(y, z \mid x)}{f_{Z \mid X}\left(z_{0} \mid x\right)} d y d z d F_{X}(x) \\
& =E\left[\frac{g(Y) \cdot 1\left(Z=z_{0}\right)}{f_{Z \mid X}\left(z_{0} \mid X\right)}\right]=E\left[\frac{g(Y) \cdot(1-D) \cdot 1\left(Z=z_{0}\right)}{f_{Z \mid X}\left(z_{0} \mid X\right)}\right]
\end{aligned}
$$

Starting from Theorem 1 and using the just derived result and the fact that the event $1\left(Z=z_{0}\right)$. $D=1$ occurs with probability zero by Assumption 1(i), one can now derive an expression for $E\left[g\left(Y^{0}\right) \mid D=1\right]$ by appropriate weighting of the observations.

$$
\begin{aligned}
E\left[g\left(Y^{0}\right) \mid D=1\right] & =\int \frac{E\left[g(Y) \mid X, Z=z_{0}\right]-E[g(Y) \cdot(1-D) \mid X]}{\operatorname{Pr}(D=1)} \cdot d F_{X} \\
& =\frac{1}{\operatorname{Pr}(D=1)} \int E\left[g(Y) \cdot(1-D) \frac{1\left(Z=z_{0}\right)}{f_{Z \mid X}\left(z_{0} \mid X\right)}\right]-E[g(Y) \cdot(1-D) \mid X] \cdot d F_{X} \\
& =\frac{1}{\operatorname{Pr}(D=1)} E\left[g(Y) \cdot(1-D) \frac{1\left(Z=z_{0}\right)-f_{Z \mid X}\left(z_{0} \mid X\right)}{f_{Z \mid X}\left(z_{0} \mid X\right)}\right] .
\end{aligned}
$$

\section{A.5 Proof of Corollary 5}

Note that $E\left[Y^{1} \mid D=1\right]=\frac{E[Y \cdot D]}{\operatorname{Pr}(D=1)}$ and apply theorem 2 with $g(Y)=Y$.

\section{A.6 Proof of Corollary 6}

By definition, $Q_{Y^{d} \mid D=1}^{\tau}=\underset{q_{1}}{\arg \min } E\left[\rho_{\tau}\left(Y^{d}-q_{1}\right) \mid D=1\right]$. We obtain the result for $Q_{Y^{1} \mid D=1}^{\tau}$ by noting that $Y^{1}=Y$ for the treated subsample. We obtain the result for $Q_{Y^{0} \mid D=1}^{\tau}$ by applying theorem 2 with $g(Y)=\rho_{\tau}(Y-q)$.

\section{A.7 Proof of Corollary 7}

For any value $z \in \operatorname{Supp}(Z)$ and a real measurable and absolutely integrable function $g$ we have

$$
\begin{aligned}
& E\left[g(Y) \mid X, R=1, Z=z_{0}\right] \\
= & E\left[g(Y) \mid X, R=1, Z=z_{0}, D=0\right]=E\left[g(Y) \mid X, Z=z_{0}, D=0\right] \\
= & E\left[g\left(Y^{0}\right) \mid X, Z=z_{0}, D=0\right]=E\left[g\left(Y^{0}\right) \mid X, Z=z_{0}\right]=E\left[g\left(Y^{0}\right) \mid X, Z=z\right] \\
= & E\left[g\left(Y^{0}\right) \mid X, Z=z, D=1\right] \operatorname{Pr}(D=1 \mid X, Z=z)+E\left[g\left(Y^{0}\right) \mid X, Z=z, D=0\right] \operatorname{Pr}(D=0 \mid X, Z=z) . \\
& E[g(Y) \mid X, R=1, Z=z, D=1]=E[g(Y) \mid X, Z=z, D=1]=E\left[g\left(Y^{1}\right) \mid X, Z=z, D=1\right] \\
& E[g(Y) \mid X, R=1, Z=z, D=0]=E[g(Y) \mid X, Z=z, D=0]=E\left[g\left(Y^{0}\right) \mid X, Z=z, D=0\right] .
\end{aligned}
$$


Combining these results we obtain

$$
\begin{aligned}
& E\left[g\left(Y^{0}\right) \mid D=1\right] \\
= & \int E\left[g\left(Y^{0}\right) \mid X, Z, D=1\right] \cdot d F_{X, Z \mid D=1} \\
= & \int \frac{E\left[g(Y) \mid X, R=1, Z=z_{0}\right]-E[g(Y) \mid X, R=1, Z, D=0] \operatorname{Pr}(D=0 \mid X, Z)}{\operatorname{Pr}(D=1 \mid X, Z)} \cdot d F_{X, Z \mid D=1} \\
= & \int \frac{E\left[g(Y) \mid X, R=1, Z=z_{0}\right]-E[g(Y) \mid X, R=1, Z, D=0] \operatorname{Pr}(D=0 \mid X, Z)}{\operatorname{Pr}(D=1)} \cdot d F_{X, Z}
\end{aligned}
$$

and

$$
\begin{aligned}
& E\left[g\left(Y^{1}\right) \mid D=1\right]=\int E[g(Y) \mid X, R=1, Z, D=1] \cdot d F_{X, Z \mid D=1} \\
= & \int \frac{E[g(Y) \mid X, R=1, Z, D=1] \operatorname{Pr}(D=1 \mid X, Z)}{\operatorname{Pr}(D=1)} \cdot d F_{X, Z} .
\end{aligned}
$$

\section{A.8 Proof of Corollary 8}

For any value $z \in \operatorname{Supp}(Z)$ and a real measurable and absolutely integrable function $g$ we have

$$
\begin{aligned}
& E\left[g(Y) \mid X, R=1, Z=z_{0}, D_{z}=1\right] \\
= & E\left[g(Y) \mid X, Z=z_{0}, D_{z}=1\right]=E\left[g\left(Y^{0}\right) \mid X, Z=z_{0}, D_{z}=1\right] \\
= & E\left[g\left(Y^{0}\right) \mid X, Z=z, D_{z}=1\right]=E\left[g\left(Y^{0}\right) \mid X, Z=z, D=1\right]
\end{aligned}
$$

and analogously

$$
E\left[g(Y) \mid X, R=1, Z=z_{0}, D_{z}=0\right]=E\left[g\left(Y^{0}\right) \mid X, Z=z, D=0\right] .
$$

It follows that

$$
\begin{aligned}
& E\left[g(Y) \mid X, R=1, Z=z_{0}\right] \\
= & E\left[g(Y) \mid X, R=1, Z=z_{0}, D_{z}=1\right] \cdot \operatorname{Pr}\left(D_{z}=1 \mid X, R=1, Z=z_{0}\right) \\
& +E\left[g(Y) \mid X, R=1, Z=z_{0}, D_{z}=0\right] \cdot \operatorname{Pr}\left(D_{z}=0 \mid X, R=1, Z=z_{0}\right) \\
= & E\left[g\left(Y^{0}\right) \mid X, Z=z, D=1\right] \cdot \operatorname{Pr}\left(D_{z}=1 \mid X, R=1, Z=z_{0}\right) \\
& +E\left[g\left(Y^{0}\right) \mid X, Z=z, D=0\right] \cdot \operatorname{Pr}\left(D_{z}=0 \mid X, R=1, Z=z_{0}\right) .
\end{aligned}
$$

We also note that

$$
\begin{aligned}
E[g(Y) \mid X, R=1, Z=z, D=1] & =E\left[g(Y) \mid X, R=1, Z=z, D_{z}=1\right] \\
& =E\left[g(Y) \mid X, Z=z, D_{z}=1\right] \\
& =E\left[g\left(Y^{1}\right) \mid X, Z=z, D=1\right]
\end{aligned}
$$

and analogously

$$
E[g(Y) \mid X, R=1, Z=z, D=0]=E\left[g\left(Y^{0}\right) \mid X, Z=z, D=0\right] .
$$


Furthermore, by Bayes' formula

$$
\begin{aligned}
& \operatorname{Pr}\left(D_{z}=0 \mid X, R=1, Z=z_{0}\right) \\
= & \frac{\operatorname{Pr}\left(R=1 \mid X, D_{z}=0, Z=z_{0}\right) \operatorname{Pr}\left(D_{z}=0 \mid X, Z=z_{0}\right)}{\operatorname{Pr}\left(R=1 \mid X, Z=z_{0}\right)} \\
= & \frac{\operatorname{Pr}\left(R=1 \mid X, D_{z}=0, Z=z\right) \operatorname{Pr}\left(D_{z}=0 \mid X, Z=z\right)}{E\left[R \mid X, Z=z_{0}\right]} \\
= & \frac{\operatorname{Pr}(R=1 \mid X, D=0, Z=z) \operatorname{Pr}(D=0 \mid X, Z=z)}{E\left[R \mid X, Z=z_{0}\right]}=\frac{E[R(1-D) \mid X, Z=z]}{E\left[R \mid X, Z=z_{0}\right]} .
\end{aligned}
$$

Combining these results we obtain after a few calculations:

$$
\begin{aligned}
& E\left[g\left(Y^{0}\right) \mid D=1\right] \\
= & \int E\left[g\left(Y^{0}\right) \mid X, Z, D=1\right] \cdot d F_{X, Z \mid D=1} \\
= & \int \frac{E\left[g(Y) \mid X, R=1, Z=z_{0}\right]-E\left[g\left(Y^{0}\right) \mid X, Z, D=0\right] \cdot \operatorname{Pr}\left(D_{z}=0 \mid X, R=1, Z=z_{0}\right)}{\operatorname{Pr}\left(D_{z}=1 \mid X, R=1, Z=z_{0}\right)} \cdot d F_{X, Z \mid D=1} \\
= & \int \frac{E\left[g(Y) R \mid X, Z=z_{0}\right]-E[g(Y) R(1-D) \mid X, Z]}{E\left[R \mid X, Z=z_{0}\right]-E[R(1-D) \mid X, Z]} \cdot d F_{X, Z \mid D=1} .
\end{aligned}
$$

and

$$
\begin{aligned}
& E\left[g\left(Y^{1}\right) \mid D=1\right] \\
= & \int E[g(Y) \mid X, Z, R=1, D=1] \cdot d F_{X, Z \mid D=1} .
\end{aligned}
$$

\section{A.9 Proof of Corollary 9}

The calculations are the same as in the previous proof up to (10).

Furthermore, by Bayes' formula

$$
\begin{aligned}
& \operatorname{Pr}\left(D_{z}=1 \mid X, R=1, Z=z_{0}\right) \\
= & \frac{\operatorname{Pr}\left(R=1 \mid X, D_{z}=1, Z=z_{0}\right) \operatorname{Pr}\left(D_{z}=1 \mid X, Z=z_{0}\right)}{\operatorname{Pr}\left(R=1 \mid X, Z=z_{0}\right)} \\
= & \frac{\operatorname{Pr}\left(R=1 \mid X, D_{z}=1, Z=z\right) \operatorname{Pr}\left(D_{z}=1 \mid X, Z=z\right)}{E\left[R \mid X, Z=z_{0}\right]} \\
= & \frac{\operatorname{Pr}(R=1 \mid X, D=1, Z=z) \operatorname{Pr}(D=1 \mid X, Z=z)}{E\left[R \mid X, Z=z_{0}\right]}=\frac{E[R D \mid X, Z=z]}{E\left[R \mid X, Z=z_{0}\right]} .
\end{aligned}
$$


Combining these results we obtain after a few calculations:

$$
\begin{aligned}
& E\left[g\left(Y^{0}\right) \mid D=1\right] \\
= & \int E\left[g\left(Y^{0}\right) \mid X, Z, D=1\right] \cdot d F_{X, Z \mid D=1} \\
= & \int \frac{E\left[g(Y) \mid X, R=1, Z=z_{0}\right]-E\left[g\left(Y^{0}\right) \mid X, Z, D=0\right] \cdot \operatorname{Pr}\left(D_{z}=0 \mid X, R=1, Z=z_{0}\right)}{\operatorname{Pr}\left(D_{z}=1 \mid X, R=1, Z=z_{0}\right)} \cdot d F_{X, Z \mid D=1} \\
= & \int \frac{E\left[g(Y) R \mid X, Z=z_{0}\right]-E[g(Y) \mid X, Z, R=1, D=0] \cdot\left(E\left[R \mid X, Z=z_{0}\right]-E[R D \mid X, Z]\right)}{E[R D \mid X, Z]} \cdot d F_{X, Z \mid D=1} \\
= & \int \frac{E\left[g(Y) R \mid X, Z=z_{0}\right]-E[g(Y) \mid X, Z, R=1, D=0] \cdot\left(E\left[R \mid X, Z=z_{0}\right]-E[R D \mid X, Z]\right)}{E[R \mid X, Z, D=1] \operatorname{Pr}(D=1)} \cdot d F_{X, Z}
\end{aligned}
$$

